\title{
On the Limit for the Representation by the Sum of Two Abundant Numbers
}

\author{
By
}

Sin Hitotumatu

\section{Introduction}

For a positive integer $n$, let us denote by $S(n)$ the sum of all divisors of $n$ including 1 and $n$ itself. If $S(n)>2 n,=2 n$, or $<2 n, n$ is called the abundant, perfect, or deficient number, respectively. We shall denote by $\mathbf{S}$ the set of all abundant numbers. It has long been known that the natural density $\delta(\mathbf{S})$ of the set $\mathbf{S}$ exists and satisfies

$$
0.241<\delta(\mathbf{S})<0.314
$$

and $\mathbf{S}$ constitutes an asymptotic basis of finite order, namely, every sufficiently large integer is a sum of a bounded number of abundant numbers. ${ }^{1)}$ Here, the abundant numbers are so defined as to include the perfect numbers, without affecting, however, the density of the set of abundant numbers.

Now, L. Moser has proved ${ }^{2)}$ that every sufficiently large integer $n>n_{0}$ can be represented as the sum of two abundant numbers, viz.

$$
n=x+y, \quad x, y \in \mathbf{S}
$$

The limit may be taken, for example, $n_{0}=83160$. For simplicity, we put

$$
\mathbf{M}=\{n \mid n=x+y, \quad x, y \in \mathbf{S}\}
$$

Received October 22, 1971.

1) cf. [3], Satz 9, p. 20. The author wishes to express his gratitude for the referee who has indicated this fact.

2) The author knows the result in the book by Ogilvy [1]. 
According to Ogilvy's book $[1]$, the precise limit $n_{0}$

$$
\min \left[n_{0} \mid\left\{n>n_{0}\right\} \subset \mathbf{M}\right]
$$

is not known. In the present paper, the author would like to show that the precise limit $n_{0}$ is 20161 .

Though some part of the proof is due to a systematic search by computer, careful conversational considerations were necessary during the search, ${ }^{3)}$ so that, I hope, it will be worthwhile to report the present result.

\section{Elementary Properties of Abundant Number}

It is well known that if $n$ is decomposed into prime factors

$$
n=p_{1}^{e_{1}} \cdots p_{m}^{e_{m}} \quad\left(p_{i} \text { 's are all distinct }\right)
$$

then we have

$$
\frac{S(n)}{n}=\prod_{i=1}^{m}\left(1+\frac{1}{p_{i}}+\cdots+\frac{1}{p_{i}^{e_{i}}}\right)
$$

Hence, if $n \in \mathbf{S}$, all multiple of $n$ is again in $\mathbf{S}$. If $n$ is a perfect number, then all its multiple other than $n$ itself is in $\mathbf{S}$. Further, if $p$ is a prime and not a Mersenne number, $2^{m-1} p \in \mathrm{S}$, provided that $2^{m}>p$. For example, every multiple of 6 except 6 , every multiple of 28 except 28 , and every multiple of $20,88, \ldots$ are all abundant numbers.

For even integers, the limit in the Moser's result is very simple. Every even integer $n \geqq 48$ can be represented by one of the forms

$$
12+6 m, \quad 20+6 m, \quad \text { or } \quad 40+6 m \quad(m \geqq 2)
$$

and hence it is in $\mathbf{M}$. On the other hand, 46 is not in $\mathbf{M}$.

The least odd abundant number is $945=3^{3} \cdot 5 \cdot 7$, and the next smallest one is $1575=3^{2} \cdot 5^{2} \cdot 7$. Combining the multiples of 6,20 or 28 (except 6

3) The main part of the present paper has been published in [2], in Japanese. 
and 28 itself) with them, it is easy to see that every integer $n$ divisible by 3,5 , or 7 is in $\mathbf{M}$ if $n>951,1555$, or 1603 respectively, and that these are the precise limit for the multiples of 3,5 , or 7 respectively.

\section{A Reduction of the Limit}

Moser's limit 83160 is equal to $945 \times 88$; here 945 is the least odd abundant number, and 88 is the least abundant number coprime to it. Hence every integer $n>83160$ can be written in the form

$$
n=945 l+88 m \quad(l, m>0)
$$

and both terms in (3) are in $\mathbf{S}$.

Now, let us remark that though $315=3^{2} \cdot 5 \cdot 7$ itself is a deficient number, it is quite close to $\mathbf{S}$, say

$$
\frac{S(315)}{315}=2\left(1-\frac{1}{105}\right)
$$

It is easy to see that $315 l \in S$ provided that $l$ contains at least one prime factor less than 105, and especially for $l=2, \ldots, 89$. Therefore, every integer $n$ written in the form

$$
n=315 l+88 m \quad(l=2, \ldots, 89 ; m>0)
$$

is in $\mathbf{M}$, and this is true for $n$ greater than

$$
315 \times 89+88=28123
$$

Thus we could reduce the limit quite a lot.

\section{Determination of the Precise Limit}

After the limit is reduced to (5), our main interest is restricted to odd integers $n$ less than (5) which is not a multiple of 3,5 , or 7 and cannot be represented as (4). They are the numbers of the form 
$315 l-88 m \quad(l=89,87, \ldots$ (odd); $m>0)$. We fix $m$ and look for the smallest odd $l_{0}$ such that

$$
t_{0}=315\left(l_{0}-3\right)-88 m \in \mathbf{S} .
$$

If we can find such $l_{0}<89$, then every $n=315 l-88 m \quad\left(l_{0} \leqq l \leqq 89\right.$, $l$ odd) may be represented as

$$
n=t_{0}+315\left(l-l_{0}+3\right) \in \mathbf{M} \text {. }
$$

The results by computer ${ }^{4)}$ for the search of $l_{0}$ is given in Table 1 . Here we have omitted the case when $m$ is divisible by 3,5 , or 7 , since the precise limit may be estimated about 20000, and we have already seen that the multiples of 3,5 , or 7 in such region are all in $\mathbf{M}$. In the Table, $u$ is the limit $315 \times 89-88 \mathrm{~m}$, and - means that there is no $l_{0}$ with (6) in the region $l_{0} \leqq 89$.

From Table 1, the only exceptional cases are $m=16$ and 68 . For such $m, n=315 l-88 m$ cannot be represented in the form $315 \times s+x$ ( $s$ odd, $x \in \mathbf{S}$ ). It is not difficult to see that all odd abundant numbers $s$ less than $26627=315 \times 89-88 \times 16$ and not a multiple of 315 are given in Table 2.

We check for each $n=315 l-88 m \quad(l=89,87, \ldots$ (odd); $m=16,68)$ whether it is represented as $n=s+t$, where $s$ is in Table 2 , and $t$ is an even abundant number. The results for a possible decomposition are given in Table 3 ; - means that no such representation is possible. Through the considerations up to here, we can conclude that 20161 is the greatest number not in M. Further, we have verified that only three numbers, say

$$
\text { 20161, 19067, } 18437
$$

are not in $\mathbf{M}$ in the region $n \geqq 18000$.

By similar check, I saw that the next largest integers not in $\mathbf{M}$ are 17891, 17261

4) In my experience, in the computation of $S(n)$, it was faster to divide $n$ by 2,3 , $4, \cdots$ up to $\sqrt{n}$ and add the divisor and the quotient when it divides $n$, than to decompose $n$ into prime factors and use the formula (2). 
both correspond to $m=58$ in Table 1 , and these are only integers not in $M$ in the region $n \geqq 17000$.

Table 1

$u=$ upper bound $315 \times 89-88 m$

$t_{0}=315\left(l_{0}-3\right)-88 m \in \mathbf{S}$

\begin{tabular}{rccc|cccc}
\hline \hline$m$ & $u$ & $l_{0}$ & $t_{0}$ & $m$ & $u$ & $l_{0}$ & $t_{0}$ \\
\hline 1 & 27947 & 11 & 2432 & 61 & 22667 & 35 & 4712 \\
2 & 27859 & 19 & 4864 & 62 & 22579 & 35 & 4624 \\
4 & 27683 & 27 & 7208 & 64 & 22403 & 43 & 6968 \\
8 & 27331 & 47 & 13156 & 67 & 22139 & 27 & 1664 \\
11 & 27067 & 27 & 6592 & 68 & 22051 & - & - \\
13 & 26191 & 11 & 1376 & 71 & 21787 & 27 & 1312 \\
16 & 26627 & - & - & 73 & 21611 & 47 & 7436 \\
17 & 26539 & 51 & 13624 & 74 & 21523 & 59 & 11128 \\
19 & 26363 & 27 & 5888 & 76 & 21347 & 35 & 3392 \\
22 & 26099 & 27 & 5624 & 79 & 21083 & 27 & 608 \\
23 & 26011 & 19 & 3016 & 82 & 20819 & 51 & 7904 \\
26 & 25747 & 19 & 2752 & 83 & 20731 & 59 & 10336 \\
29 & 25483 & 59 & 15088 & 86 & 20467 & 43 & 5032 \\
31 & 25307 & 47 & 11132 & 88 & 20291 & 67 & 12416 \\
32 & 26219 & 59 & 14824 & 89 & 20203 & 75 & 14848 \\
34 & 25043 & 47 & 10868 & 92 & 19939 & 35 & 1984 \\
37 & 24779 & 43 & 9344 & 94 & 19763 & 51 & 6848 \\
38 & 24691 & 19 & 1696 & 97 & 19499 & 75 & 14144 \\
41 & 24427 & 27 & 3952 & 101 & 19147 & 43 & 3712 \\
43 & 24251 & 27 & 3776 & 103 & 18971 & 43 & 3536 \\
44 & 24163 & 35 & 6208 & 104 & 18883 & 35 & 928 \\
46 & 23987 & 19 & 992 & 106 & 18707 & 83 & 15872 \\
47 & 23899 & 43 & 8464 & 107 & 18619 & 51 & 5704 \\
52 & 23459 & 19 & 464 & 109 & 18443 & 43 & 3008 \\
53 & 23371 & 43 & 7936 & 113 & 18091 & 59 & 7696 \\
58 & 22931 & 75 & 17576 & 116 & 17727 & 43 & 2392 \\
59 & 22843 & 27 & 2368 & & & & \\
\hline & & & & & & &
\end{tabular}


Table 2

\begin{tabular}{rlll}
\hline \multicolumn{1}{c}{$s$} & prime factors & \multicolumn{1}{c}{$s$} & \multicolumn{1}{c}{ prime factors } \\
\hline 5775 & $3 \cdot 5^{2} \cdot 7 \cdot 11$ & 15015 & $3 \cdot 5 \cdot 7 \cdot 11 \cdot 13$ \\
6435 & $3^{2} \cdot 5 \cdot 11 \cdot 13$ & 19305 & $3^{3} \cdot 5 \cdot 11 \cdot 13$ \\
6825 & $3 \cdot 5^{2} \cdot 7 \cdot 13$ & 19635 & $3 \cdot 5 \cdot 7 \cdot 11 \cdot 17$ \\
7425 & $3^{3} \cdot 5^{2} \cdot 11$ & 21945 & $3 \cdot 5 \cdot 7 \cdot 11 \cdot 19$ \\
8085 & $3 \cdot 5 \cdot 7^{2} \cdot 11$ & 22275 & $3^{4} \cdot 5^{2} \cdot 11$ \\
8415 & $3^{2} \cdot 5 \cdot 11 \cdot 17$ & 23205 & $3 \cdot 5 \cdot 7 \cdot 13 \cdot 17$ \\
8925 & $3 \cdot 5^{2} \cdot 7 \cdot 17$ & 25245 & $3^{3} \cdot 5 \cdot 11 \cdot 17$ \\
9555 & $3 \cdot 5 \cdot 7^{2} \cdot 13$ & 25935 & $3 \cdot 5 \cdot 7 \cdot 13 \cdot 19$ \\
12705 & $3 \cdot 5 \cdot 7 \cdot 11^{2}$ & 26565 & $3 \cdot 5 \cdot 7 \cdot 11 \cdot 23$ \\
\hline
\end{tabular}

Table 3

\begin{tabular}{|c|c|c|c|c|}
\hline \multirow[b]{2}{*}{$l$} & \multicolumn{2}{|c|}{$m=16$} & \multicolumn{2}{|c|}{$m=68$} \\
\hline & $n$ & decomposition & $n$ & decomposition \\
\hline 89 & 26627 & $9555+17072$ & 22051 & $6435+15616$ \\
\hline 87 & 25997 & $8925+17072$ & 21421 & $8925+12496$ \\
\hline 85 & 25367 & $8415+16952$ & 20791 & $8415+12376$ \\
\hline 83 & 24737 & $12705+12032$ & 20161 & - \\
\hline 81 & 24107 & $19635+4472$ & 19531 & $8415+11116$ \\
\hline 79 & 23477 & $8085+15392$ & 18901 & $8085+10816$ \\
\hline 77 & 22847 & $5775+17072$ & 18271 & $5775+12496$ \\
\hline 75 & 22217 & $6825+15392$ & 17641 & $6825+10816$ \\
\hline 73 & 21587 & $9555+12032$ & 17011 & $8415+8596$ \\
\hline 71 & 20957 & $8925+12032$ & 16381 & $8085+8296$ \\
\hline 69 & 20327 & $5775+14552$ & & $\cdots \cdots$ \\
\hline 67 & 19697 & $7425+12272$ & & \\
\hline 65 & 19067 & - & & \\
\hline 63 & 18437 & - & & \\
\hline 61 & 17807 & $5775+12032$ & & \\
\hline 59 & 17177 & $12705+4472$ & & \\
\hline
\end{tabular}

\section{References}

[1] Ogilvy, C.S., Tomorrow's Math.: Unsolved problems for the amateur, Oxford Univ. Press, 1962.

[2] Hitotumatu, S., On the Moser's problem to represent an integer by the sum of two abundant numbers (in Japanese), Sugaku 24, No.2, 1972.

[3] Ostmann, H.-H., Additive Zahlentheorie, II, Erg. d. Math. u. ihrer Grenzgeb. N.F. 11, Springer-Verlag, 1956. 